\title{
Über die Bereiche der Normalwerte der Serumproteinfraktionen in Abhängigkeit vom Kollektiv
}

\author{
Von U. B. SeIfFert und S. Gerhardt \\ Kliniscb-Chemiscbe Laboratorien des Zentrums der Inneren Mediqin \\ (Direktorium: Prof. Dr.J. Frey, Prof. Dr. K. Schöffling, Prof. Dr. W. Siede, Prof. Dr. Martin) \\ Universität Frankfurt/M.
}

(Eingegangen am 9. November 1970)

\begin{abstract}
Die Normalbereiche der Serumproteinfraktionen, ermittelt mit Hilfe der Mikroelcktrophorese auf Celluloseacetatfolien, wurden für drei verschiedene Kollektive untersucht.

1. Blutspender und Berufstätige baben einen um 6,4\% (3,9 rel.\%) höheren Albuminwert als ambulante Patienten. Diese Albuminerniedrigung wird durch erhöhte Werte der drei Globulinfraktionen ausgeglichen. Dieser Befund bleibt auch nach Aufgliederung der Kollektive nach Altersgruppen erhalten. Diese Beobachtung wird nicht durch pathologische Werte der Patienten hervorgerufen, da diese eliminiert wurden und vor allem die Standardabweichung $s$ bei den drei Kollektiven in gleicher Größenordnung ist.

2. Die Werte der Proteinfraktionen zeigen eine statistisch gesicherte Altersabhängigkeit. Diese Beobachtung ist bei den drei Kollektiven gleich. Mit zunehmendem Alter der Probanden wird der Albumingehalt geringer. Die niedrigen Werte werden hauptsächlich durch eine Erhöhung der $\boldsymbol{\gamma}$-Globulinwerte ausgeglichen.

3. Weibliche Probanden haben niedrigere Albuminwerte als männliche Probanden. Diese Abweichung ist zwar statistisch gesichert, aber nur von geringem Ausmaß.

Auf die Bedeutung dieser Befunde für die Beurteilung der Elektrophorese in der klinisch-chemischen Diagnostik wird hingewiesen.
\end{abstract}

\section{The ranges of normal values for serum protein fractions and their dependence on collective}

The normal ranges of values for serum protein fractions, determined by microelectrophoresis on cellulose acetate strips were studied for three different collectives.

1. The albumin values for blood donors and employed persons is $6.4 \%$ ( 3.9 rel. $\%$ ) higher than for ambulant patients. This low albumin level is balanced by increased values for the three globulin fractions. These findings still hold if the collectives are subdivided into age groups; they do not result from pathological values in the patients, since these were eliminated and, moreover, the standard deviation is of the same order for the three collectives.

2. The values for the protein fractions show a statistically confirmed age-dependence. This observation is the same for all three collectives. The albumin level decreases with increasing age of the probands. These lower values are balanced chiefly by an increase in the $\gamma$-globulin values.

3. Female probands have lower albumin values than males. Although this difference is statistically significant, it is only slight.

These findings should be considered evaluating the electrophoresis for the purpose of diagnosis.

Die elektrophoretische Serumproteinfraktionierung wird mit Vorteil auf Celluloseacetatfolie anstatt auf Papier durchgeführt (1-4). Im Vordergrund stehen dabei die bessere Reproduzierbarkeit und größere Analysengenauigkeit. Nach Einführung der neuen Methode im klinischen Laboratorium fiel auf, daß die Normalbereiche verschiedener Kollektive erheblich voneinander abweichen. Für die Beurteilung der Ergebnisse der Elektrophorese ist es aber unerläßlich, die Ursachen für diese Abweichungen zu kennen. Daher wurden drei verschiedene Kollektive systematisch durchuntersucht und die Ergebnisse miteinander verglichen.

Ein Vergleich der in der Literatur mitgeteilten Normalwerte zeigt ebenfalls Abweichungen voneinander (4 bis 7). Als eine der Ursachen für diese Abweichungen wurde das unterschiedliche Alter der untersuchten Probanden angenommen (8, 9). Mit zunehmendem Alter der untersuchten Probanden kommt es zu einer Abnahme des Albumingehalts im Serum verbunden mit einer relativen Zunahme aller Globulinfraktionen.
Dieser Befund blieb nicht unwidersprochen (10). Die Autoren konnten auch keine Angaben darüber machen, welche der Globulinfraktionen relativ vermehrt wird, wenn der Albumingehalt absinkt. Über den Einfluß des Geschlechts der untersuchten Probanden auf die Werte der Serumproteinfraktionen bestehen nur widersprüchliche Meinungen $(4,8)$. Andere Faktoren, die die Normalbereiche der Proteinfraktionen wesentlich beeinflussen, sind nicht bekannt.

\section{Material und Methoden}

Die elektrophoretische Auftrennung der Serumproteine wurde nach dem Verfahren der Firma Beckman Instruments (USA) auf Celluloseacetatfolien durchgeführt. Als Puffer diente 0,075 M Natriumbarbiturat $\mathrm{pH} 8,6$. Die Trennzeit betrug $20 \mathrm{Min}$. bei $250 \mathrm{~V}=2,0 \mathrm{~V} / \mathrm{cm}$. Die Folie wurde mit Poinceau $\mathrm{S}$ gefärbt und mit Hilfe einer Lösung aus $25 \mathrm{~m} /$ Eisessig und $75 \mathrm{~m} /$ Methanol transparent gemacht. Die so erhaltenen Folien wurden mit dem für Celluloseacetatfolien adaptierten Beckman Analytrol 2 ausgewertct. Monitrol 2 der Firma Dade diente als Kontrollserum zur Qualitätskontrolle. Das Auswertegcrät (Analytrol) vurde nach den Angaben des Kontrollserums Monitrol 2 geeicht. Bei 
dieser Einstellung ergab eine Mischung zu gleichen Teilen von gereinigtem Albumin und $\gamma$-Globulin der Firma Serva, Heidelberg in einer Konzentration von insgesamt $8 \mathrm{~g} / 100 \mathrm{~m} l$ nach elektrophotetischer Auftrennung ein Verhältnis von Albumin zu $\gamma$ Globulin wie 1,0:0,885. Zur Untersuchung gelangte Serum. Das Gesamteiweiß wurde mit der Biuretmethode bestimmt. Der analytische Fehler der gesamten Methode am Kontrollserum gemessen beträgt für Albumin $V=4,6 \%$

Das Kollektiv I waren Personen, die in der Klinik durchuntersucht wurden. Ein großer Teil dieser Personen waren ambulante Patienten $(\mathrm{n}=1000)$.

Das Kollektiv II waren Personen aus einem Verwaltungsbetrieb, die während der Untersuchung ihrer Arbeit nachgingen und sich gesund fühlten $(n=235)$.

Das Kollektiv III waren Blutspender, meistens männliche Probanden $(n=170)$.

\section{Statistische Auswertung}

Der Verteilungstyp der Meßwerte wurde durch graphische Darstellung der in Klassen gruppierten Meßwerte ermittelt. Dabei ergab sich eine Gauss'sche Normalverteilung. Aus diesem Grunde konnten der Mittelwert $\overline{\mathrm{X}}$ und die Standardabweichung beziehungsweise der Bereich der Normalwerte mit Hilfe von Wahrscheinlichkeitspapier ermittelt werden. Die prozentuale kumulative Häufigkeit der Stichproben je Klasse wurde in dem Wahrscheinlichkeitspapier eingetragen. Aus der erhaltenen Geraden konnte der Mittelwert sowie die obere und die untere 2s Grenze direkt abgelesen werden. Bei den Geraden wichen die obeten Werte

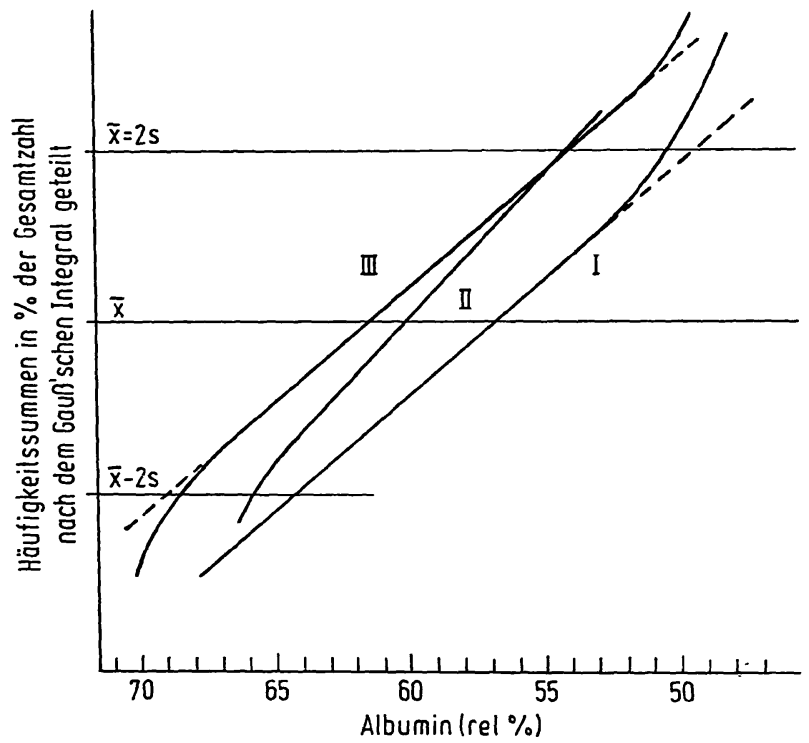

Abb. 1

Graphische Darstellung der Ermittlung der Normalbereiche auf Wahrscheinlichkeitspapier. Die Abszisse ist linear, die Ordinate nach dem Gauss'schen Integral unterteilt. Gestrichelt ist die Anpassung an eine Gerade zur Elimination der im Kollektiv enthaltenen pathologischen Werte gezeichnet. Der Mittelwert, die obere $(97,3 \%)$ und die untere $(2,7 \%) 2$ s-Grenze sind durch horizontale Linien gekennzeichnet. Kollektiv I $\mathrm{I}$ - Patienten, II = Berufstätige, III = Blutspender mehr oder weniger von dem geradlinigen Kurvenverlauf ab. Diese Abweichung wurde durch die in dem Kollektiv enthaltenen pathologischen Werte verursacht. Durch die Verlängerung der Geraden nach oben wurden die pathologischen Werte eliminiert $(11,12,15)$ (Abb. 1). Aus den erhaltenen Werten wurde die Signifikanz $\mathrm{p}$ exrechnet. Die Unterschiede der erhaltenen Meßwerte wurden mit Hilfe des $t$-Tests errechnet und aus diesem Wert die Signifikanz p $(11, .12,15)$.

\section{Ergebnisse}

Der Proteingehalt der zur Untersuchung gelangten Serumproben zeigt bei den drei untersuchten Kollektiven keine signifikanten Unterschiede. Es ist daher möglich, die Meßergebnisse der Proteinfraktionierung in Relativprozenten anzugeben, statt in absoluten Eiweißwerten.

Die Untersuchungsergebnisse der drei untersuchten Kollektive sind in Tabelle 1 wiedergegeben. Besonders auffällig sind die Unterschiede in der Höhe des Albuminanteiles. Bei dem Kollektiv der Blutspender wird ein Mittelwert von 60,4 rel.\% gefunden, bei den berufstätigen Personen ein Mittelwert von 59,1 rel. \%, während der Albuminwert des Patientenkollektivs bei 56,5 rel. $\%$ liegt. Die Differenz zwischen den Blutspendern und dem Patientenkollektiv beträgt 3,9 rel.\% $(\mathrm{p}=0,001)$. Die Albuminerniedrigung bei $\operatorname{dem} \mathrm{Pa}-$ tientenkollektiv führt zu einer relativen Erhöhung der gesamten Globulinfraktion. Es läßt sich so nicht sagen, welche der Globulinfraktionen erhöht ist. Es ist auffällig, daß die Blutspender eine recht hohe $\beta$-Globulinfraktion aufweisen.

Um eine Erklärung für die oben gemachten Beobachtungen zu erhalten, werden die einzelnen Kollektive nach Altersgruppen geordnet und untersucht. Die erhaltenen Ergebnisse sind in Tabelle 2 zusammengefaßt. Die in Tabelle 1 gezeigten Unterschiede zwischen den drei Kollektiven bleiben unabhängig von der Altersgruppierung erhalten. Bei allen drei Kollektiven zeigen aber die Werte der Proteinfraktionen eine Abhängigkeit vom Alter. Der Albuminwert hat in der Gruppe der jüngsten Probanden den höchsten Wert. Mit zunehmendem Alter fällt der Albumingehalt ab. Dieses Ergebnis ist bei allen drei Kollektiven statistisch gesichert $(p=0,011)$. Der relative Anstieg der Globuline teilt sich nach der Altersgruppierung folgendermaßen auf. Für die $\alpha_{1}$ - und $\alpha_{2}$-Fraktionen läßt sich bei keinem Kollektiv eine statistisch $\mathrm{zu}$ sichernde $\mathrm{Zu}$ nahme mit dem Alter ermitteln. Die $\beta$-Fraktion zeigt

Tab. 1

Vergleich der Mittelwerte und Standardabweichungen der Eiweißfraktionen der Kollektive I $=$ Patienten, II $=$ Berufștätige, II $\dot{I}=$ Blutspender

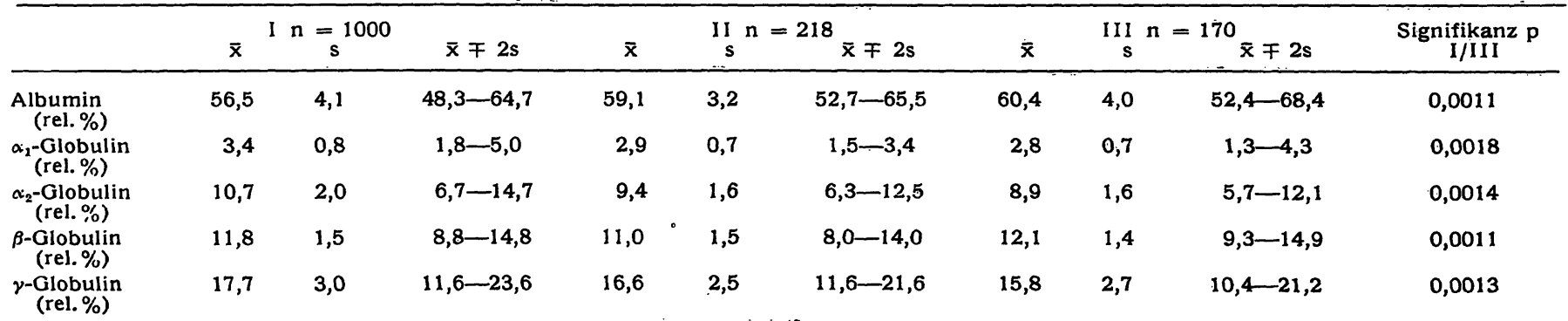


Tab. 2

Abhängigkeit der Proteinfraktionen vom Alter der Probanden

\begin{tabular}{|c|c|c|c|c|c|c|c|c|c|}
\hline \multirow{2}{*}{$\begin{array}{c}\text { Alter der } \\
\text { Probanden } \\
\text { (Jahre) }\end{array}$} & \multicolumn{3}{|c|}{ Albumin $\bar{x}($ rel. $\%)$} & \multicolumn{3}{|c|}{$a_{1}$-Globulin $\bar{x}($ rel. $\%)$} & \multicolumn{3}{|c|}{$\alpha_{2}$-Globulin $\bar{x}($ rel. $\%)$} \\
\hline & 1 & II & 111 & 1 & II & III & 1 & 11 & I I I \\
\hline \multirow[t]{2}{*}{$\begin{array}{r}19 \\
20-29 \\
30-39 \\
40-49 \\
50-59 \\
60\end{array}$} & $\begin{array}{l}\mathbf{5 8 , 6} \\
\mathbf{5 7 , 9} \\
\mathbf{5 7 , 1} \\
\mathbf{5 6 , 9} \\
\mathbf{5 5 , 8} \\
\mathbf{5 4 , 7}\end{array}$ & $\begin{array}{l}60,4 \\
60,0 \\
59,8 \\
59,2 \\
57,2\end{array}$ & $\begin{array}{c}(64,2) \\
61,7 \\
60,1 \\
59,5 \\
59,3 \\
(58,1)\end{array}$ & $\begin{array}{l}3,2 \\
3,5 \\
3,3 \\
3,4 \\
3,4 \\
3,7\end{array}$ & $\begin{array}{l}3,3 \\
3,0 \\
3,0 \\
2,9 \\
3,1\end{array}$ & $\begin{array}{c}(2,4) \\
2,7 \\
2,7 \\
3,0 \\
3,1 \\
(3,0)\end{array}$ & $\begin{array}{l}10,9 \\
11,0 \\
10,3 \\
10,4 \\
10,5 \\
11,2\end{array}$ & $\begin{array}{r}9,8 \\
9,7 \\
9.5 \\
9,5 \\
10,2\end{array}$ & $\begin{array}{c}(7,1) \\
8,9 \\
8,7 \\
9,3 \\
8,9 \\
(9,3)\end{array}$ \\
\hline & I & II & 111 & I & II & III & I & II & III \\
\hline $\begin{array}{r}19 \\
20-29 \\
30-39 \\
40-49 \\
50-59 \\
60\end{array}$ & $\begin{array}{l}10,9 \\
11,3 \\
11,7 \\
11,7 \\
11,9 \\
12,2\end{array}$ & $\begin{array}{l}10,4 \\
10,8 \\
11,4 \\
11,7 \\
11,2\end{array}$ & $\begin{array}{c}(11,7) \\
11,9 \\
12,2 \\
12,2 \\
12,6 \\
(12,2)\end{array}$ & $\begin{array}{l}16,3 \\
16,3 \\
17,6 \\
17,6 \\
18,4 \\
18,3\end{array}$ & $\begin{array}{l}16,1 \\
16,7 \\
16,4 \\
16,8 \\
17,6\end{array}$ & $\begin{array}{l}(14,6) \\
15,0 \\
16.2 \\
16,3 \\
16,2 \\
(17,4)\end{array}$ & $\begin{array}{r}68 \\
215 \\
214 \\
262 \\
235 \\
211\end{array}$ & $\begin{array}{l}14 \\
57 \\
51 \\
55 \\
31\end{array}$ & $\begin{array}{l}3 \\
63 \\
42 \\
40 \\
17 \\
3\end{array}$ \\
\hline
\end{tabular}

Tab. 3

Abhängigkeit der Proteinfraktionen vom Alter und Geschlecht der Probanden

\begin{tabular}{|c|c|c|c|c|c|c|c|c|}
\hline $\begin{array}{l}\text { Alter der } \\
\text { Probanden }\end{array}$ & $\begin{array}{c}1 \\
\sigma^{\prime}\end{array}$ & $\begin{array}{l}\text { Alb } \\
1 \\
O\end{array}$ & $\begin{array}{c}1 . \%) \\
\text { II } \\
0^{\circ}\end{array}$ & $\begin{array}{l}\text { II } \\
\text { Q }\end{array}$ & $\frac{1}{j}$ & $\begin{array}{l}1 \\
0\end{array}$ & $\begin{array}{l}11 \\
\mathrm{r}^{\circ}\end{array}$ & $\begin{array}{l}\text { II } \\
\text { Q }\end{array}$ \\
\hline $\begin{array}{r}19 \\
20-29 \\
30-39 \\
40-49 \\
50-59 \\
60\end{array}$ & $\begin{array}{l}\mathbf{5 8 , 7} \\
\mathbf{5 7 , 3} \\
\mathbf{5 7 , 4} \\
\mathbf{5 6}, 1 \\
\mathbf{5 5 , 2} \\
\mathbf{5 4 , 3}\end{array}$ & $\begin{array}{l}57,3 \\
56,7 \\
55,3 \\
55,9 \\
54,6 \\
54,5\end{array}$ & $\begin{array}{l}61,3 \\
60,1 \\
60,2 \\
57,6\end{array}$ & $\begin{array}{l}60,0 \\
59,6 \\
59,5 \\
58,0 \\
58,0\end{array}$ & $\begin{array}{l}14 \\
43 \\
46 \\
48 \\
47 \\
34\end{array}$ & $\begin{array}{l}14 \\
36 \\
37 \\
36 \\
45 \\
42\end{array}$ & $\begin{array}{l}13 \\
27 \\
31 \\
14 .\end{array}$ & $\begin{array}{l}13 \\
44 \\
24 \\
24 \\
17\end{array}$ \\
\hline
\end{tabular}

bei allen drei Kollektiven eine deutliche Zunahme mit dem Alter $(p=0,02)$. Die $\gamma$-Globulinfraktion zeigt die größte Altersabhängigkeit. Die Zunahme ist besonders ausgeprägt nach dem 50. Lebensjahr $(p=0,016)$.

Die Ergebnisse der Aufgliederung der Kollektive I und II nach dem Geschlecht sind in Tabelle 3 zusammengefaßt. Bei dieser Untersuchung zeigt sich, daß die weiblichen Probanden einen signifikant niedrigeren Albuminwert haben als die männlichen. Die Unterschiede der erhaltenen Werte der Globulinfraktionen sind nicht signifikant.

\section{Diskussion}

Die je nach Kollektiv unterschiedliche Höhe der Albuminkonzentration wird durch eine relative $\mathrm{Zu}$ nahme der gesamten Globulinfraktion ausgeglichen. Dieser Befund ist am schwierigsten zu deuten, eine sichere Erklärung kann nicht abgegeben werden. Es erscheint unwahrscheinlich, daß die gemachten Beobachtungen methodisch bedingt sind. Wie in Abbildung 1 ersichtlich, gehen bei der graphischen Ermittlung der Mittelwerte und der Streuung pathologische Werte nicht in die Resultate ein. Durch die Anpassung der Kurve an eine Gerade werden die pathologischen Werte eliminiert $(11,12,15)$. Das trifft nicht nur für das Kollektiv I zu, sondern auch für die anderen beiden Kollektive. Der Anteil der eliminierten pathologischen Werte ist bei dem Kollektiv der Patienten am größten. Würde man trotzdem annehmen, daß die Unterschiede der Albuminwerte durch geringfügig pathologisch vexänderte Werte hervorgerufen werden, dann müßte die. Streuung $s$ beim Kollektiv I größer sein als beim Kollektiv III. Dies ist aber nicht der Fall; wie in Abbildung 1 veranschaulicht, verlaufen die Geraden von Kollektiv I und III parallel. Es erscheint daher unwahrscheinlich, daß die beobachtete Abweichung durch pathologische Werte verursacht wird.

Die höheren Albuminwerte des Blutspenderkollektivs könnten die Folge einer besseren körperlichen Kondition beziehungsweise vermehrter körperlicher $\mathrm{Be}$ wegung sein. Eine derartige Beobachtung bei sportlich trainierten Personen wurde in der Literatur bisher nicht gemacht. Keliner und Heinde (13) haben die Auswirkung von Blutspenden auf die Serumproteine näher untersucht. Sie haben gefunden, daß unmittelbar nach der Blutspende eine Hypoalbuminämie vorliegt, während nach 2 Monaten ein höherer Albuminwert beobachtet wurde. Diese Autoren deuten den Befund als eine überschießende Regeneration. $\mathrm{Da}$ es sich bei dem Kollektiv III lediglich um Gelegenheitsspender handelt, erscheint diese Erklärung wenig plausibel. Die Annahme, daß der körperliche Trainingszustand einen Einfluß auf den Albuminspiegel nimmt, findet Unterstützung darin, daß der Albumingehalt des Serums bei dem Kollektiv I sehr niedrig liegt.

Das Absinken der Albumine sowie die Erhöhung der Globuline mit zunehmendem Alter wurde schon beschrieben. Nach SIEDE (14) handelt es sich um ein Zeichen einer allgemeinen Beeinträchtigung der Leberfunktion. Auch andere, in der Leber synthetisierte Stoffwechselprodukte sind von dem Altersprozeß betroffen. Für die Beurteilung der klinischen Elektrophorese ist es aber wichtig zu wissen, welche der Globulinfraktionen kompensatorisch ansteigt, wenn die Albumine mit zunehmendem Alter absinken. In dieser Studie konnte eindeutig gezeigt werden, daß die $\beta$ - 
Globuline ansteigen, besonders aber die Fraktion der $\gamma$-Globuline. Der Anstieg der $\beta$-Globulinfraktion dürfte wohl mit der Vermehrung der Lipoproteine in engem Zusammenhang stehen.

Der Unterschied zwischen männlichen und weiblichen Probanden zeigt sich nur deutlich im Albumingehalt, ist aber so gering, daß dieser Unterschied bei der
Beurteilung der Elektrophorese wohl kaum ins Gewicht fallen dürfte. Die hier ermittelten Veränderungen der Proteinfraktionen des Serums zeigen deutlich, wie vorsichtig die Elektrophorese in der klinisch-chemischen Diagnostik beurteilt werden muß. Außerdem zeigt diese Studie, wie kritisch das Kollektiv zur Ermittlung, von Normalwerten ausgesucht werden muß.

\section{Literatur}

1. Albert-Recht, F., Clin. chim. Acta (Amsterdam) 4, 627 (1959). - 2. Busch, O., Klin. Wschr. 37, 758 (1959). - 3. Busch, O. und J. TAMm, Klin. Wschr. 39, 143 (1961). - 4. Fessed, W. J., Arch. internal Med. 547 (1964). - 5. KapLAN, A. und J. SavorY, Clin. Chem. (New York) 11, 937 (1965). - 6. Demaret, M., Ann. biol. clin., Paris 24, 369 (1966). - 7. Shepard, H. und B. W. Coye, Amer. J. Clin. Path. 43, 464 (1965). - 8. Nöcker, J. und H. BeMrm, Zschr. Altersforsch. 9, 3 (1955). -9. LuetsCher, J. A., Physiol. Rev., Baltimore 27, 621 (1947). - 10. LYoN, B. und H. A. Новаск, Geriatrics, Minneapolis (1961). - 11. LINDER,
A., „Statistische Methoden“, Verlag Birkhäuser, Basel (1951). 12. SACHS, L., „Statistische Auswertungsmethoden“, Springer Berlin, Heidelberg, New York (1968). - 13. KELLNER, W. und K. Heindr, Ärztl. Wschr. 155 (1954). - 14. Siede, W., in Handbuch der praktischen Geriatrie, Krankheiten der Leber. Hrsg. von W. Doberauer und A. Hittmair, Bd. II. Verlag Enke, Stuttgart (1967). - 15. Rohrberg, A., Die Anwendung der Wahrscheinlichkeits- und Häufigkeitsnetze. Hrsg. von C. Schleicher \& Schüll, Dassel Kr. Einbeck (1967).

Priv.-D'oz. Dr. Ulrich B. Seiffert Leiter der Klinischen Laboratorien im ZIM 6 Frankfurt/M.

Ludwig-Rehn-Str. 14 